\title{
SIR2 and SIR4 interactions differ in core and extended telomeric heterochromatin in yeast
}

\author{
Sabine Strahl-Bolsinger, ${ }^{1}$ Andreas Hecht, ${ }^{2}$ Kunheng Luo, and Michael Grunstein ${ }^{3}$ \\ Department of Biological Chemistry and School of Medicine and the Molecular Biology Institute, University of California, \\ Los Angeles, California 90095 USA
}

\begin{abstract}
Yeast core telomeric heterochromatin can silence adjacent genes and requires RAP1, SIR2, SIR3, and SIR4 and histones $\mathrm{H} 3$ and $\mathrm{H} 4$ for this telomere position effect. SIR3 overproduction can extend the silenced domain. We examine here the nature of these multiprotein complexes. SIR2 and SIR4 were immunoprecipitated from whole-cell extracts. In addition, using formaldehyde cross-linking we have mapped SIR2, SIR4, and RAP1 along telomeric chromatin before and after SIR3 overexpression. Our data demonstrate that SIR2 and SIR4 interact in a protein complex and that SIR2, SIR3, SIR4, and RAP1 map to the same sites along telomeric heterochromatin in wild-type cells. However, when overexpressed, SIR3 spreads along the chromosome and its interactions are dominant to those of SIR4 and especially SIR2, whose detection is decreased in extended heterochromatin. RAP1 binding at the core region is unaffected by SIR3 overproduction and RAP1 shows no evidence of spreading. Thus, we propose that the structure of core telomeric heterochromatin differs from that extended by SIR3.
\end{abstract}

[Key Words: Heterochromatin; telomeres; silencing; SIR proteins; RAP1]

Received September 25, 1996; revised version accepted November 15, 1996.

Heterochromatin was cytologically defined as that fraction of the eukaryotic genome that is constitutively condensed throughout the cell cycle (Heitz 1928). Such regions, often found near centromeres or telomeres, can repress adjacent genes epigenetically. For example, euchromatic genes placed adjacent to centromeric heterochromatin in Drosophila melanogaster are repressed in some but not all cells. This silencing is inherited clonally, resulting in a mosaic phenotype that is referred to as position effect variegation $(\mathrm{PEV}$; for review, see Henikoff 1990). PEV has provided a tool for the identification of a number of suppressors or enhancers of variegation that exhibit dosage effects. As a result, it has been proposed that heterochromatin involves the nucleation of multimeric protein complexes that can then spread into adjacent euchromatic regions (Locke et al. 1988). However, despite the identification of a variety of transacting factors that affect PEV (for review, see Weiler and Wakimoto 1995), the molecular basis for heterochromatin formation and propagation in Drosophila has been elusive.

The yeasts also have chromosomal regions with fea-

Present addresses: ${ }^{1}$ Lehrstuhl für Zellbiologie und Pfalanzenphysiologie, Universität Regensburg, D-93040 Regensburg, Germany; ${ }^{2}$ Max-PlanckInstitute for Immunobiology, Department of Molecular Embryology,

D-79108 Freiburg, Germany.

${ }^{3}$ Corresponding author.

E-MAIL mg@ewald.mbi.ucla.edu; FAX (310) 206-9073. tures of heterochromatin (Thompson et al. 1993; Allshire et al. 1994). In Saccharomyces cerevisiae these are near telomeres and at the silent mating loci ( $H M L \alpha$ and $H M R a$ ) where they can repress heterologous genes in an epigenetic manner (Gottschling et al. 1990; Laurenson and Rine 1992). In wild-type cells, telomeric position effect (TPE) extends some $2-4 \mathrm{~kb}$ toward the centromere (Gottschling et al. 1990). A number of proteins (RAP1, SIR2, SIR3, and SIR4, as well as histones $\mathrm{H} 3$ and H4) have been identified that are involved in both forms of silencing (Aparicio et al. 1991; Kurtz and Shore 1991; Liu et al. 1994; Thompson et al. 1994b). Of these, overexpression of the limiting protein SIR3 causes TPE to spread as much as $16-20 \mathrm{~kb}$ or more (Renauld et al. 1993). RAP1 is the only factor that interacts with specific DNA sequences, in particular telomeric $\mathrm{C}_{1-3} \mathrm{~A}$ repeats and silencer DNA elements adjacent to the HM loci (Shore and Nasmyth 1987; Buchman et al. 1988). Because RAP1 interacts with SIR3 and SIR4 in the yeast two-hybrid system, and at least with SIR3 in vitro, it has been suggested that RAP1 may recruit SIR3 and SIR4, thus nucleating the heterochromatic structure (Moretti et al. 1994). In vitro GST pull-down experiments have also shown interactions between SIR3 and SIR4 and the amino termini of histones $\mathrm{H} 3$ and $\mathrm{H} 4$ at those histone regions involved in silencing in vivo. Hence it has been proposed that after interaction at RAP1 sites, these SIR proteins polymerize along chromatin by interacting with the histone tails (Hecht et al. 1995). 
This model is supported by coimmunolocalization studies demonstrating the presence of RAP1, SIR3, and SIR4 in foci formed by the association of telomeres (Palladino et al. 1993; Gotta et al. 1996). Also, RAP1, SIR3, SIR4, and histones are found in complexes immunoprecipitated from whole-cell extracts (Hecht et al. 1996), supporting and extending earlier work showing the coimmunoprecipitation of RAP1 and SIR4 (Cockell et al. 1995). Moreover, immunoprecipitation of SIR3 in combination with formaldehyde cross-linking has mapped SIR3 to the silent HM and telomeric loci. These data show that SIR3 spreads in a histone H4-dependent manner approximately as far as silencing extends even when SIR3 is overexpressed (Hecht et al. 1996). Unexpectedly, although RAP1 and SIR3 interact in vitro in the absence of histones, an $\mathrm{H} 4$ amino-terminal mutation in vivo prevents this interaction. Conversely, a deletion of the RAP1 domain involved in silencing affects SIR3-H4 interactions. These data argue that RAP1-SIR3/SIR4 interactions are stabilized by the presence of nucleosomes. This is further supported by genetic data showing that suppression of histone $\mathrm{H} 4$ silencing defects by the SIR3 mutation SIR3N205 (Johnson et al. 1990) requires the carboxyl terminus of RAP1 (Liu and Lustig 1996).

The roles of SIR 2 and SIR4 in the initiation and spreading of heterochromatin have been a mystery. SIR2 is an evolutionarily conserved protein (Brachmann et al. 1995) that also suppresses rDNA recombination /Gottlieb and Esposito 1989). Moreover, both disruption and overexpression of SIR2 affects general histone acetylation levels (Braunstein et al. 1993). However, the link between telomeric silencing and rDNA recombination is presently unknown and it is uncertain whether the effects on acetylation are direct. SIR4 has very limited homology to nuclear lamins (Diffley and Stillman 1989); however, it has not been demonstrated as yet that there is a functional conservation. Although SIR2 and SIR4 are both required for extended TPE when SIR3 is overexpressed (Renauld et al. 1993), it is unclear whether they are both structural proteins of the heterochromatic complex in vivo and whether they both spread with SIR3 during the extension of heterochromatin.

To address these questions we have immunoprecipitated SIR2 and SIR4 from whole-cell extracts. In addition, using formaldehyde cross-linking we have mapped the presence of SIR2, SIR4, and RAP1 along telomeric heterochromatin before and after SIR3 overexpression. Our data suggest that core telomeric heterochromatin in wild-type cells differs in structure from extended telomeric heterochromatin produced by SIR3 overexpression.

\section{Results}

SIR2 coimmunoprecipitates with SIR3 and SIR4 from yeast whole-cell extracts

We have shown that SIR3 coimmunoprecipitates SIR4, RAP1, and the four core histones $\mathrm{H} 2 \mathrm{~A}, \mathrm{H} 2 \mathrm{~B}, \mathrm{H} 3$, and $\mathrm{H} 4$ from yeast whole-cell extracts (Hecht et al. 1996). To determine whether SIR2 is also present in the SIR3associated complex or complexes, hemagglutinin (HA) epitope-tagged SIR3 (SIR3HA) was expressed in the strain AYH2.8. SIR3HA, which functions normally in all aspects of silencing examined (Hecht et al. 1996), was immunoprecipitated from whole-cell extracts using monoclonal antibody (17D09) directed against the HA epitope (Wilson et al. 1984). Western blot analyses show that both SIR4 and SIR2 coimmunoprecipitate with SIR3HA (Fig. 1A, lane 3) in a manner dependent on the HA tag (Fig. 1A, lane 1). This occurs equally even after extensive DNase I digestion (data not shown), thus ruling out indirect DNA-mediated interactions. Therefore, SIR2 is a member of the SIR3-associated protein complex.

\section{SIR2-SIR4 and SIR4-SIR3 protein complexes}

To determine whether interactions between pairwise
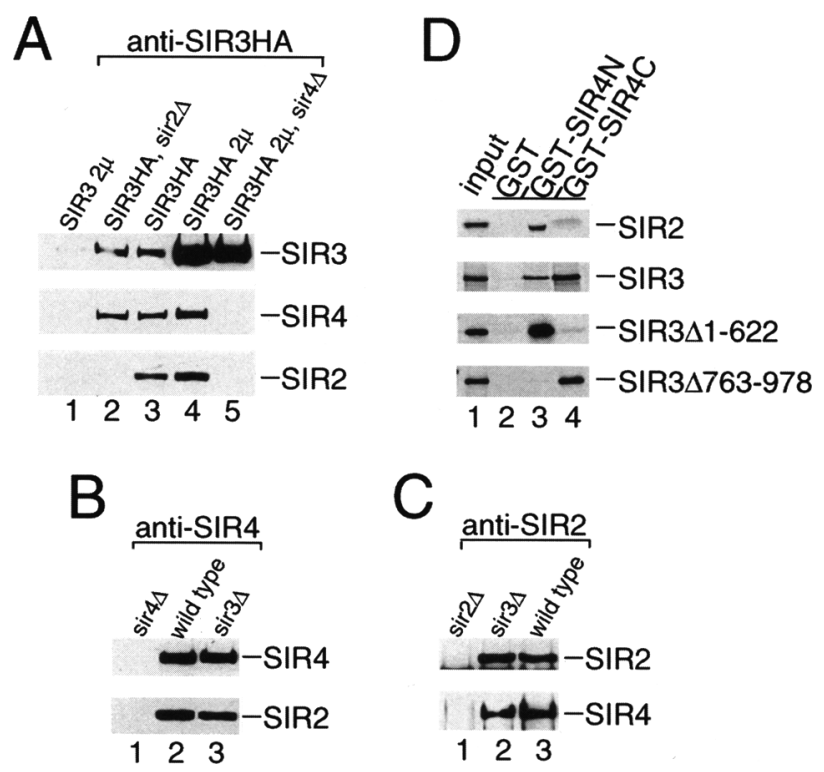

Figure 1. Interactions between SIR3, SIR4, and SIR2 in wholecell extracts and in vitro. $(A)$ SIR3HA was immunoprecipitated, using a monoclonal anti-HA antibody (17D09), from whole-cell

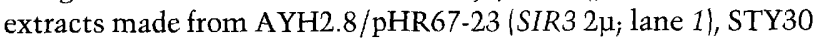
(SIR3HA; sir2A; lane 2), AYH2.45 (SIR3HA; lane 3), AYH2.8)

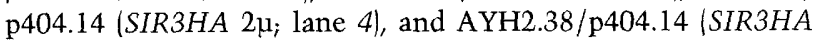
$2 \mu$; sir4 $\Delta$; lane 5). (B) SIR4 was precipitated using affinity-purified anti-SIR4 polyclonal antibodies from whole-cell extracts made from AYH2.38/p404.14 (lane 1), AYH2.45 (lane 2), and AYH2.8/pRS424 (sir3 $\Delta$; lane 3). (C) SIR2 was precipitated using affinity-purified anti-SIR2 polyclonal antibodies from wholecell extracts made from STY30 (lane 1), AYH2.8/pRS424 (lane 2), and AYH2.45 (lane 3). $(A-C)$ Immunoprecipitates were analyzed by SDS-PAGE and Western blot sequentially probed with affinity-purified anti-SIR2, -SIR3, and -SIR4 antibodies. $(D)$ Direct SIR2-SIR4 and SIR4-SIR3 interactions. In vitro-produced $\left[{ }^{35} \mathrm{~S}\right]$ methionine-labeled full-length SIR2, SIR3, and the deletion mutants SIR3A 1-622 and SIR3A 763-978 (fractions of the input material are shown in lane 1) were incubated with GST (lane 2), GST-SIR4N (amino acids 142-591; lane 3), or GST-SIR4C (amino acids 1144-1358; lane 4). Interacting proteins were eluted and analyzed by SDS-PAGE and fluorography as described in Materials and Methods. 
combinations of SIR proteins require the presence of the remaining SIR protein, SIR3HA was immunoprecipitated from strains wild type or deleted for SIR2, SIR3, or SIR4. Because overexpression of SIR3 extends the silencing complex, we also examined the effect of SIR3HA overexpression on these interactions. In analogous experiments (Fig. 1B,C) SIR4 and SIR2 were immunoprecipitated using affinity purified polyclonal antibodies (see Materials and Methods for details). The precipitates were probed for each of the SIR proteins by Western blotting. When SIR3HA is expressed on a multicopy plasmid, its levels increase $\sim 10$ - to 15 -fold whereas SIR2 and SIR4 levels show only a minor increase as compared with levels in the wild-type control strain (Fig. 1A, cf. lanes 3 and 4). When antibodies to either SIR4 (Fig. 1B, lanes 2 and 3) or SIR2 (Fig. 1C, lanes 3 and 2) are used for immunoprecipitation, SIR2 and SIR4 interact even when SIR3 is absent. Because deletion of SIR4 abolishes the coprecipitation of SIR2 by SIR3 (Fig. 1A, lane 5) and deletion of SIR2 does not affect SIR3 interactions with SIR4 (Fig. 1A, lane 2), these data argue for SIR2-SIR4 and SIR4-SIR3 protein interactions in the immunoprecipitates.

\section{Direct SIR2-SIR4 and SIR4-SIR3 interactions in vitro}

To address the question of direct interactions between SIR2, SIR3, and SIR4 we used an in vitro protein-affinity assay. Intragenic trans-complementation of SIR4 mutations has suggested that both the amino and carboxyl termini mediate SIR4 functions (Marshall et al. 1987). SIR3-SIR4 interactions have been detected in yeast twohybrid studies using the SIR4 carboxy-terminal amino acids 1204-1358 (Moretti et al. 1994). Therefore, aminoand carboxy-terminal regions of SIR4 were fused to glutathione $S$-transferase $(\mathrm{GST})$; the hybrid proteins were expressed and purified from Escherichia coli strain BL21, and immobilized on glutathione-Sepharose beads. The coding sequences of full-length SIR2, SIR3, and deletion constructs of SIR3 were transcribed and translated in vitro in the presence of $\left[{ }^{35} \mathrm{~S}\right]$ methionine. GST-immobilized proteins were incubated with the labeled proteins, and the bound proteins were eluted, resolved by SDSPAGE, and detected by fluorography. We found that fulllength SIR 2 binds directly to the amino terminus of SIR 4 (amino acids 142-591) GST-SIR4N (Fig. 1D, lane 3) much more strongly than to the SIR 4 carboxyl terminus (amino acids 1144-1358) GST-SIR4C (Fig. 1D, lane 4). Full-length SIR3 interacts with both GST-SIR4N and GST-SIR4C, but binding to the carboxyl terminus is more pronounced. SIR3 deletion mutants (SIR3 1 1-622 and SIR3 $463-978$ ) that bind either to GST-SIR4N or GST-SIR4C show that full-length SIR3 can interact with both amino- and carboxy-terminal regions of SIR4.

\section{SIR2 and SIR4 are associated with core telomeric heterochromatin in vivo}

Our recent work has identified SIR3 as a structural component of yeast heterochromatin, present at $H M R a$, $H M L \alpha$, and telomeres. Moreover, SIR3 has been shown to spread from core telomeric heterochromatin (up to $2-4 \mathrm{~kb}$ from the telomere) to at least $17.5 \mathrm{~kb}$ (Hecht et al. 1996) in extended telomeric heterochromatin when SIR3 is overexpressed. To assess whether SIR2 and SIR4 are also associated with these heterochromatic regions in vivo SIR2, SIR4, and SIR3HA were each immunoprecipitated from the strain AYH2.45 after in situ cross-linking with formaldehyde. Chromatin was sonicated to an average fragment size of $0.5-1 \mathrm{~kb}$ as described (Hecht et al. 1996). SIR2-, SIR4-, and SIR3-associated DNA was analyzed by PCR. To distinguish between silent and expressed chromatin we used three different sets of genespecific primer pairs (schematically shown in Fig. 2A). The first set of primer pairs compares the silent mating type loci $H M R a$ and $H M L \alpha$ with the expressed MATa locus and the euchromatic GAL1 gene. The second set includes a telomeric copy of URA3 (URA3Tel; Gottschling et al. 1990) next to the telomere of the left arm of chromosome VII (VII-L) and URA3 at its normal locus on the left arm of chromosome V (V-L). The third set contains a region $0.77 \mathrm{~kb}$ distal from the telomere of the right arm of chromosome VI (VI-R) and the ACT1 gene, also located on chromosome VI but some $52 \mathrm{~kb}$ from the right telomere of this $270-\mathrm{kb}$ chromosome.

We found that the anti-SIR4 antibodies immunoprecipitate DNA sequences from the silent loci $H M R a$ and $H M L \alpha$ preferentially (10- to 15 -fold) as compared with the active MATa or GAL1 loci (Fig. 2B, cf. lane 2 to input material in lane 6). The silent sequences at URA3Tel and the chromosome VI-R telomere proximal region at $0.77 \mathrm{~kb}$ are also greatly enriched in comparison to sequences from the euchromatic URA3 and ACT1 regions. This association of SIR4 with silent loci requires the presence of the other SIR proteins, because it is disrupted in $\operatorname{sir} 3 \Delta$ or sir2 $\Delta$ deletion strains (Fig. 2B, lanes 3 and 4). Similarly, anti-SIR2 antibodies were used to demonstrate that SIR2 is associated with the silent loci (Fig. 2C, lane 2) as is SIR3HA (Fig. 2D, lane 2; Hecht et al. 1996). Moreover, SIR2 and SIR3 presence at silent chromatin is also greatly reduced in the absence of any of the other SIR proteins (Fig. 2C,D, lanes 3 and 4). Therefore, like SIR3 (Hecht et al. 1996), SIR2 and SIR4 are chromosomal proteins preferentially bound to the silent HM loci and telomeres in vivo. In addition, the interaction seen between SIR2, SIR3, or SIR4 with heterochromatic regions after cross-linking requires the presence of all three SIR proteins.

\section{SIR2, SIR3, and SIR4 are each associated with core telomeric heterochromatin at the same distance from the telomere}

In wild-type yeast, core TPE decreases strongly with increasing distance from the end of the chromosome. To determine whether all three SIR proteins spread as TPE spreads over this region we analyzed the distribution of SIR2, SIR3, and SIR4 along the telomere-distal region of chromosome VI-R that lacks the repetitive $\mathrm{Y}^{\prime}$ and $\mathrm{X}$ sequences (for review, see Olson 1991). SIR3HA, SIR4, and SIR2 were immunoprecipitated under cross-linking con- 
Figure 2. In vivo association of SIR4, SIR2, and SIR3 with silent chromatin in wildtype and mutant strains. Whole-cell extracts were prepared and chromatin was sonicated to an average DNA size of $0.5-1.0$ $\mathrm{kb}$ in formaldehyde cross-linked strains (Hecht et al. 1996). PCR was done with primers shown schematically in $A$. Immunoprecipitation was performed using affinity-purified anti-SIR4 $(B)$, anti-SIR2 polyclonal antibodies $(C)$, and anti-HA antibody 17 D09 against SIR3-HA (D). PCR products were resolved on $6 \%$ polyacrylamide gels. (Lanes 1-4) PCR products of DNA precipitates from strains wild type or mutant for specific SIR genes. (Lanes 5-8) PCR products from the respective input extracts. (Lanes 9-10) 2.5-fold serial dilutions of wild-type input extracts. (M) DNA size standard. The strains used were AYH2.45 (wild-type; $B-D$, lane 2), STY30 (sir2 $\Delta ; B, D$, lane 4; $C$, lane 1$)$, AYH2.8/pRS424 (sir $3 \Delta$; $B, C$, lane 3), AYH2.38/p404.14 (sir4 4 ; $B$, lane 1 , and $D$, lane 3$),$ STY36 (sir4 $4, C$, lane 4), and AYH2.8/pHR67-23 (SIR3 $2 \mu ; D$, lane 1 .

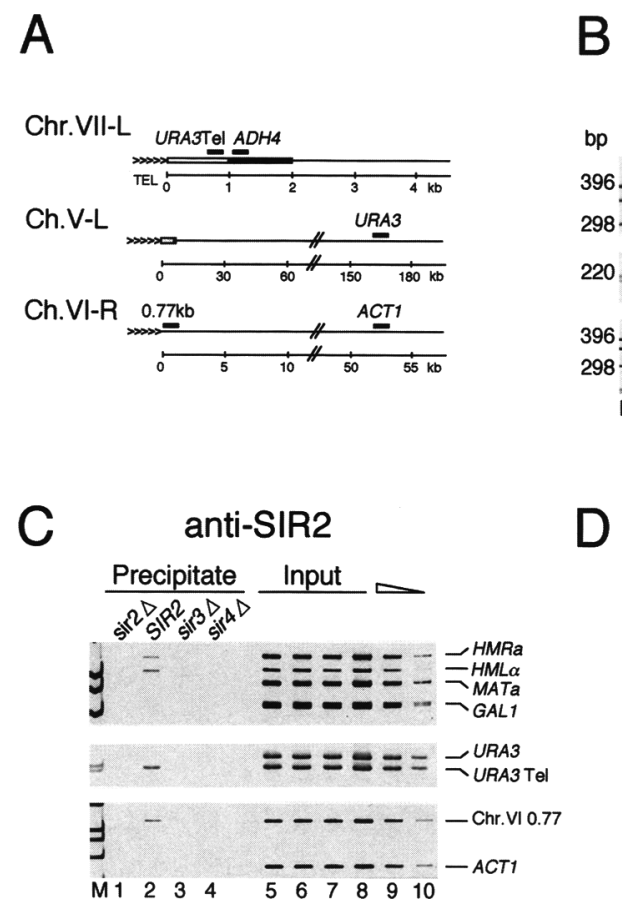

ditions from the strain AYH2.45 as described above. The chromosomal DNA was extensively sonicated, resulting in DNA fragments of which $>95 \%$ were between 0.3 and $0.5 \mathrm{~kb}$ as quantitated by Southern blots probed with either $A C T 1$ or the $0.5 \mathrm{~kb}$ region of chromosome VI-R/data not shown). This more severe sonication was done to improve distance measurements near the telomere, as mapping resolution may be affected by DNA fragment size. The precipitated DNA was analyzed by PCR using primer pairs directed against the subtelomeric region of chromosome VI-R at $0.5,1.0,1.8,2.2,2.8,5.0$, and $7.5 \mathrm{~kb}$ distal from the telomere (schematically shown in Fig. $3 \mathrm{~A})$. ACT1 served as a control for an expressed locus.

There is considerable enrichment of the silent $0.5-\mathrm{kb}$ region of chromosome VI-R as compared with the euchromatic ACT1 gene in the anti-SIR2, -SIR3HA, or -SIR4 immunoprecipitates (Fig. 3B). Moreover, the amount of telomere-distal DNA precipitated decreases rapidly with increasing distance from the telomere. While there is still a small fraction of DNA precipitated by each of the antibodies at $2.8 \mathrm{~kb}$ as compared with 0.5 $\mathrm{kb}$ from the telomere, the amount of DNA precipitated at $5.0 \mathrm{~kb}$ and $7.5 \mathrm{~kb}$ is not significantly greater than that at the ACT1 locus. When the amounts of DNA precipitated at each distance point are normalized to the amount brought down at $0.5 \mathrm{~kb}$ for each antibody, we find that the distributions of SIR2, SIR3, and SIR4 extending from the telomere at the heterochromatic core are indistinguishable (Fig. 3C).

\section{SIR4 and SIR2 interactions with extended telomeric heterochromatin are reduced}

We wished to determine the extent to which SIR2 and
SIR4 spread with SIR3 upon SIR3 overexpression. Immunoprecipitations of SIR3HA, SIR4, and SIR2 from strain AYH2.45 (expressing SIR3HA from a genomic copy of the gene) and AYH2.45/p404.14 (expressing SIR3HA from a multicopy plasmid) were performed after crosslinking with formaldehyde. The distributions of SIR4 and SIR2 in comparison to SIR3 at the HM loci, URA3Tel, and the regions adjacent to the telomere of chromosome VI-R and the right arm of chromosome V (V-R) were then analyzed (primers schematically shown in Fig. 4A). In contrast to chromosome VI-R, chromosome $\mathrm{V}-\mathrm{R}$ contains $\mathrm{X}$ and $\mathrm{Y}^{\prime}$ repetitive sequences (for review, see Olson 1991). Because of the presence of these repetitive elements, only regions further than $10 \mathrm{~kb}$ from the telomere of chromosome V-R were examined.

To correct for the ability of different SIR antibodies to precipitate different quantities of DNA, the amounts of template DNA used in the wild-type strain PCR reactions were normalized such that they result in a very similar amount of PCR product for URA3Tel (Fig. 4B, cf. lanes 2,5 , and 81 . The same fraction of template DNA was then used for the immunoprecipitates from cells overexpressing SIR3. Thus, the effects of SIR3 overexpression on SIR3, SIR4, and SIR2 spreading to telomere distal locations can be compared directly (Fig. 4B, lanes 3,6 , and 9). When SIR3 is overexpressed, it is evident that the anti-SIR3HA antibody strongly precipitates DNA as far as $15 \mathrm{~kb}$ from the telomeric end of chromosome VI-R (Fig. 4B, lane 3; Hecht et al. 1996). In contrast to antiSIR3HA, anti-SIR4 pulls down less DNA at $0.77 \mathrm{~kb}$ when SIR3HA is overexpressed in comparison to wild-type cells. However, although there is some DNA precipitated by anti-SIR 4 at telomere distal locations, the amounts of DNA are comparatively less than those 
A

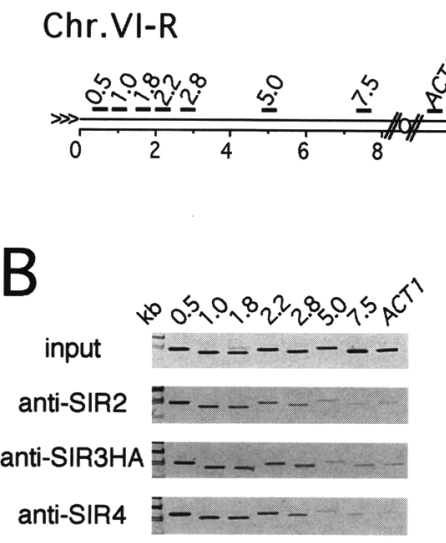

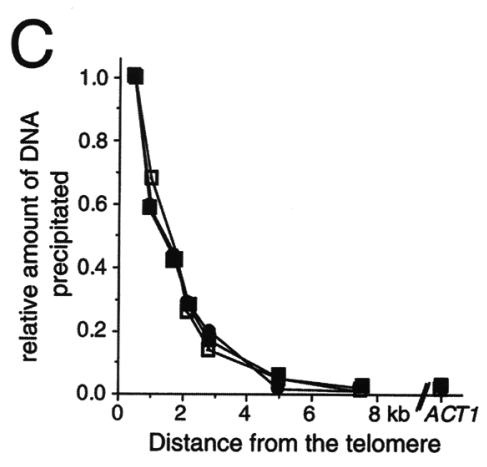
the relative abundance of 1.0. Average values from three experiments are shown. (ם) SIR2; ( ) SIR3; ( $\square$ ) SIR4. To exclude the possibility that the observed decreases in the amount of precipitated DNA with increasing distance from the telomere are a result of heterogeneity in the size of the input chromatin resulting from the chromatin fragmentation by sonication, we compared chromatin size distributions, which showed no significant differences (data not shown).

pulled down by anti-SIR3HA (Fig. 4B, lane 6). Anti-SIR2 also pulls down less DNA at $0.77 \mathrm{~kb}$ upon SIR3HA overproduction (Fig. 4B, lane 9). We observe very similar levels of anti-SIR2 precipitated DNA at telomere distal locations in strains that are deleted for SIR2 (Fig. 4B, lane 7) and in those that contain single-copy or multiple-copy SIR3HA (Fig. 4B, lanes 8 and 9). The residual binding in the sir2 $\Delta$ strain is likely due to other members of the SIR2 gene family (Brachmann et al. 1995). Therefore, these data, and those of chromosome V-R, argue that SIR3 overexpression results in the loss of SIR4 and SIR2 contacts from telomere proximal locations. There is evidence of some SIR 4 spreading along the telomere distal regions at levels lower than those observed for SIR3HA. We do not see significant SIR2 spreading under these conditions. However, we do observe very weak spreading of SIR2 after longer photographic exposure of the gels (data not shown).

\section{RAP1 does not show spreading upon SIR3 overexpression}

RAP1 is associated with the telosome that is only some $300 \mathrm{bp}$ in length and contains the $\mathrm{C}_{1-3} \mathrm{~A}$ repeats at the telomeric end (Wright et al. 1992). To ask whether RAP1 is associated only with the telosome in vivo, RAP1 was immunoprecipitated after cross-linking using affinity purified polyclonal anti-RAP1 antibodies. We analyzed the distribution of RAP1 along the telomere distal region of chromosome VI-R in the wild-type strain AYH2.45 as described above for the SIR proteins (see Fig. 3). Surprisingly, anti-RAPl precipitates DNA as far as $2-4 \mathrm{~kb}$ from the telomere in a manner similar to that of anti-SIR4 (Fig. 5A). We then asked whether RAP1 spreads along the chromosome when SIR3 is overexpressed. The strains analyzed were AYH2.45 (genomic SIR3HA) and AYH2.8/

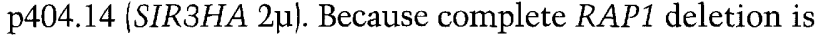
lethal, the rap1-21 mutant strain AYH2.46/p419.3 lacking the carboxy-terminal 28 amino acids of the protein required for silencing RAP1-SIR3 interaction (Liu et al. 1994) and for SIR3 association with silent chromatin (Hecht et al. 1996) was used as a control (Fig. 5B, lane 1). In the wild-type strain, we find that RAP1 is associated with $H M R a, H M L \alpha$, and telomeric regions of chromosome VII-L $(A D H 4$; see Fig. 2A) and chromosome VI-R $(0.77 \mathrm{~kb}$ and $2.5 \mathrm{~kb}$; Fig. $5 \mathrm{~B}$, lane 2). Although there is much less DNA pulled down by the anti-RAP1 antibody at 5.0 and $7.5 \mathrm{~kb}$, there is a focus of binding at $15.0 \mathrm{~kb}$. SIR3 overexpression causes no detectable changes in the binding of RAP1 at any of these regions. This is also true for chromosome V-R, which has foci of RAPl binding at $10.0,17.5$, and $30.0 \mathrm{~kb}$ unrelated to SIR3 overexpression. We conclude that extension of heterochromatin by SIR3 overexpression does not titrate RAP1 from the telomeric core. Neither does it result in detectable levels of RAP1 spreading along the chromosome.

\section{Discussion}

Our data demonstrate that SIR2 is a component of the SIR3, SIR4, and RAP1 chromatin complex in which SIR2 interacts with SIR4, which in turn interacts with SIR3. SIR2, SIR4, SIR3, and RAP1 are associated with silent chromatin in vivo and are present at the telomeric core complex at similar distances from the telomere in wildtype cells. However, when overproduction of SIR3 spreads TPE inward, SIR3 appears more abundant than SIR4 and SIR 2 in extended heterochromatin. RAP1 binding appears unchanged in the telomere proximal region and this protein shows no evidence of spreading as SIR3 is overexpressed. In light of these findings we must reevaluate the means by which telomeric heterochromatin is formed and extended along chromatin in yeast. 
A

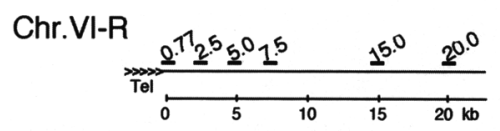

Chr.V-R

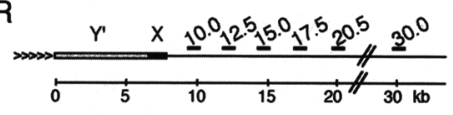

B
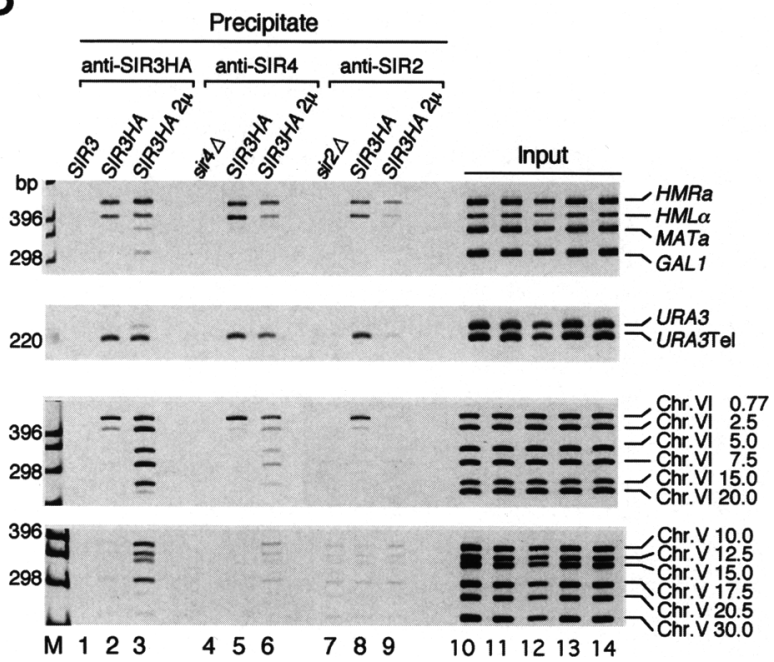

Figure 4. Limited spreading of SIR 4 and SIR2 in extended telomeric heterochromatin. Whole-cell extracts were prepared from in situ formaldehyde cross-linked strains AYH2.45 (SIR3HA, lanes 2,5,8, and 13), AYH2.45/p404.14 (SIR3HA 2u; lanes 3,6,9, and 14), AYH2.8/pHR67-23 (SIR3 2 $\mu$; lanes 1 and 10), AYH2.38/

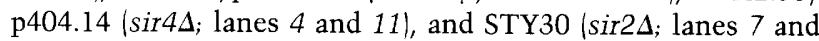
12). SIR3HA, SIR4, and SIR2 were immunoprecipitated as described in Fig. 2. DNA samples from the precipitates (lanes 1-9) and from aliquots of the whole-cell extracts (input, lanes 10-14) were analyzed by PCR with gene-specific primer pairs (schematically shown in $A$ and Fig. 2A). PCR products resolved on $6 \%$ polyacrylamide gels are shown $(B)$. (M) DNA size standard.

\section{Core telomeric heterochromatin}

The coimmunoprecipitation and in vitro protein interactions described above argue for direct contacts between SIR2 and SIR4 as well as between SIR4 and SIR3 even in the absence of DNA. These are further supported by recent data demonstrating that SIR2 and SIR3 bind to SIR4 affinity columns (Moazed and Johnson 1996). In the context of chromatin we do not see these independent SIR protein interactions. Using formaldehyde crosslinking we found that SIR2 and SIR4 are, like SIR3, present at strikingly similar distances in core telomeric heterochromatin in wild-type yeast. Moreover, mutations in $\mathrm{H} 4$ that prevent silencing do not allow SIR 3 binding to HM loci and telomeres (Hecht et al. 1996) and deletions of SIR2, SIR3, or SIR4 prevent the interaction of the entire SIR complex with silent regions. Finally, a truncated version of RAP1 (rap1-21) that is missing the car- boxy-terminal region involved in silencing but is still able to bind to the silent HM loci and telomeres prevents the binding of SIR3 to this region (Hecht et al. 1996). These data argue that although SIR2 can interact with SIR4 and SIR4 with SIR3, the interaction of SIR2-SIR4SIR3 with the telomeric core heterochromatin requires the entire SIR complex, RAPl, and the histone H4 amino terminus.

Our cross-linking data do not address the stoichiometry of SIR2-SIR4-SIR3 interactions, merely the distance from the telomeric end at which SIR proteins are present. Because RAPl is thought to bind DNA at the telosome that extends $\sim 300 \mathrm{bp}$ from the chromosomal end (Wright et al. 1992), it is surprising that RAP1 is present in the core heterochromatin at the same distances from the telosome as are the SIR proteins. This may be explained if the telosome folds back so that the RAP1-SIRhistone interactions occur at different distances in core telomeric heterochromatin (Hecht et al. 1995, 1996). An example of such a loop structure is shown schematically in Figure 6A and may include not only heterotypic but also homotypic interactions, as SIR3 and SIR4 can also self-associate (Chien et al. 1991; Moretti et al. 1994). This complex, formed of many weakly interacting partners, would become increasingly stable as more SIR proteins are recruited and if multiple telomeres form foci contributing to higher SIR protein concentrations. Several other observations support this model. First, RAP1SIR3-histone $\mathrm{H} 4$ interactions are interdependent in cell extracts (Hecht et al. 1996) and amino-terminal mutations of SIR3 that suppress the loss of silencing resulting from mutations in histone $\mathrm{H} 4$ also partially suppress silencing defects of the rap1-21 allele (Johnson et al. 1990; Liu and Lustig 1996). Second, silencing of the HM mating loci is improved near RAP1 sites at the telomeres even when those sites are separated by as much as $23 \mathrm{~kb}$ from the HM loci (Thompson et al. 1994a; Maillet et al. 1996). Third, although longer telomeres containing defective RAP1 decrease silencing frequency (Hardy et al. 1992), longer telomeres containing intact RAP1 actually improve silencing frequency over that of wild type (Kyrion et al. 1993). In each case, looping of RAPl sites into chromatin through RAP1-SIR-histone complexes may stabilize heterochromatin and improve silencing.

\section{Extended telomeric heterochromatin}

We have shown that overexpressed SIR3 spreads from the telomere along subtelomeric chromatin (Hecht et al. 1996). Given the SIR2-SIR4-SIR3 interaction, one might expect that SIR 4 and SIR2 would spread equally strongly with SIR3 as it extends inward. In fact, this is not the case. Although SIR3 interactions with chromatin remain high, SIR 4 and especially SIR2 appear to be lost from telomere proximal regions upon SIR3 overexpression (Fig. 4B). We also observe that SIR4 spreads as far but more weakly than SIR3 into telomere distal regions. SIR2 appears to spread even more weakly and we do not see spreading of RAP1. Because SIR3, SIR4, and SIR2 binding at telomere proximal regions is normalized in wild-type cells (Fig. 4B), the differences in antibody bind- 


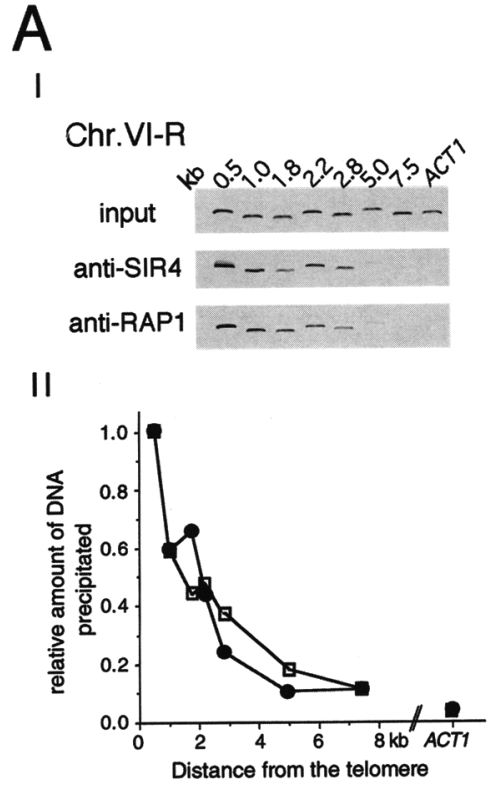

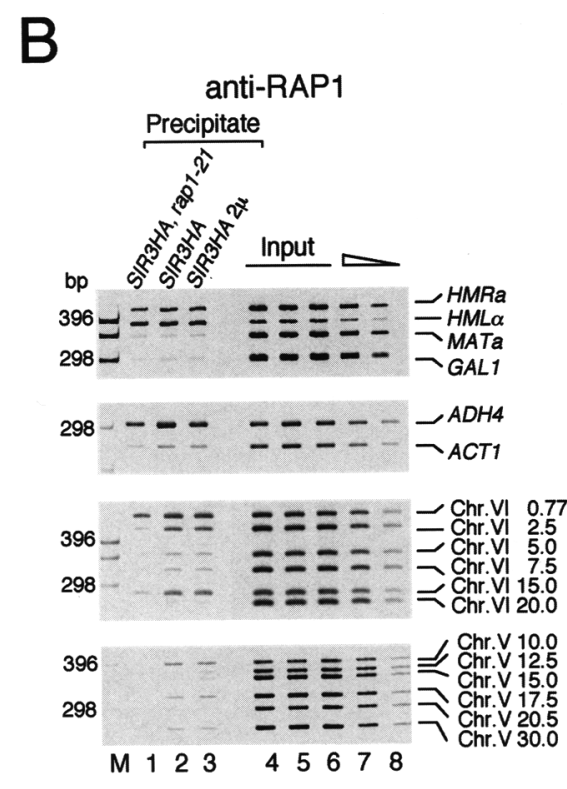

Figure 5. (A) Association of RAP1 with telomeres in wild-type cells is similar to that of SIR4. RAP1 and SIR4 were immunoprecipitated from strain AYH2.45 using affinity-purified anti-RAP1 or anti-SIR 4 polyclonal antibodies. The coprecipitated DNA was analyzed as described in Fig. 3. (I) Resulting PCR products resolved on $15 \%$ polyacrylamide gels. (II) Relative abundances of the fragments plotted against the distance from the telomere (for details, see Fig. 3); $(\mathbf{O})$ RAP1; ( $\square$ ) SIR4. (B) Overexpression of SIR3 does not alter the chromosomal distribution of RAP1. RAP1 was immunoprecipitated under cross-linking conditions from whole-cell extracts made from strain AYH2.46/p419.3 (rap1-21; SIR3HA 2u; lanes 1 and 4), AYH2.45 (SIR3HA; lanes 2 and 5), and AYH2.8/p404.14 (SIR3HA $2 \mu$; lanes 3 and 6). DNA samples from the precipitates (lanes 1-3), from aliquots of the whole-cell extracts (input; lanes 4-6), and serial dilutions thereof (lanes 7 and 8) were analyzed by PCR with gene-specific primer pairs (schematically shown in Figs. 2A and 4A). PCR products resolved on $6 \%$ polyacrylamide gels are shown. (M) DNA size standard. ing of silent loci when SIR3 is overexpressed cannot be a result of the different epitopes involved or their location in the silencing complex. It is also unlikely that overexpressed SIR3 masks SIR4 and especially SIR2 epitopes, because very similar decreases in SIR 4 binding to heterochromatin are seen when the HA epitope is fused to the amino terminus of SIR4 and immunoprecipitated (data not shown). Also, such masking should be greatest near the telomere where SIR 3 binds best, yet anti-SIR4 precipitates DNA most efficiently near the telomere (Fig. 4, lane 6). Finally, Western blot analyses of SIR4 immunoprecipitated after formaldehyde cross-linking using antiSIR4 polyclonal antibodies show no significant differences in the amount of SIR4 precipitated from wild-type and SIR 3 overexpressing strains. We conclude that SIR4 and SIR 2 proteins are considerably less abundant in extended telomeric heterochromatin that encompasses the previous core region when SIR3 is overexpressed (Fig. 6B). The greater loss of SIR2 could be attributable to, among other possibilities, the liberation and subsequent binding of this protein to the highly repetitive ribosomal DNA locus that interacts with SIR2 in cross-linking studies (S. Strahl-Bolsinger and M. Grunstein, unpubl.).

The frequency of silencing as measured by 5 -fluoroorotic acid (5-FOA) sensitivity to URA3 expression can decrease by several orders of magnitude with increasing distance from the core (Renauld et al. 1993). Is it possible that only those few DNA molecules which have the core stoichiometry of SIR2/SIR3/SIR4 are capable of silencing URA3 in heterochromatin extended by SIR3 overexpression? We have measured repression of URA3 at the telomere of chromosome $\mathrm{V}-\mathrm{R}$ (at which the $\mathrm{X}$ and $\mathrm{Y}^{\prime}$ repeated elements were removed; Renauld et al. 1993) by the more direct RNase protection assay. We find there to be a close correspondence between percent SIR3 binding and URA3 repression when normalized to maximal binding and derepression, respectively. This is true at various distances from the telomere in both core and extended telomeric heterochromatin (data not shown). Therefore, SIR3 alone or SIR3 interacting with the lesser quantities of SIR4 and SIR2 are likely to be sufficient for repression in telomere-distal heterochromatin extended by SIR3 overproduction.

These results must be reconciled with previous work demonstrating that SIR2 and SIR4 are required for the extension of TPE along with SIR3 (Renauld et al. 1993) and that the components of the silencing complex interact in a balanced fashion for an essential aspect of silencing. For example, overexpression of SIR4 or its carboxyterminal fragment decreases silencing at HM loci and telomeres and delocalizes SIR3 and RAP1 from telomeric foci (Marshall et al. 1987; Cockell et al. 1995). One possible explanation is that balanced SIR4-SIR2-SIR3 concentrations are required mainly for the nucleation of heterochromatin near RAP1-binding sites. This specific ratio between the SIR proteins may be necessary to successfully compete for RAP1 binding sites against RIF1 (Hardy et al. 1992), a negative regulator of the silencing function of RAP1 at telomeres (Kyrion et al. 1993; Buck and Shore 1995). However, this stoichiometry may be less important for the further propagation of heterochromatin. In extension, SIR3-SIR3 or SIR3histone interactions driven by higher SIR3 concentrations, even when SIR3 is on a centromeric plasmid 

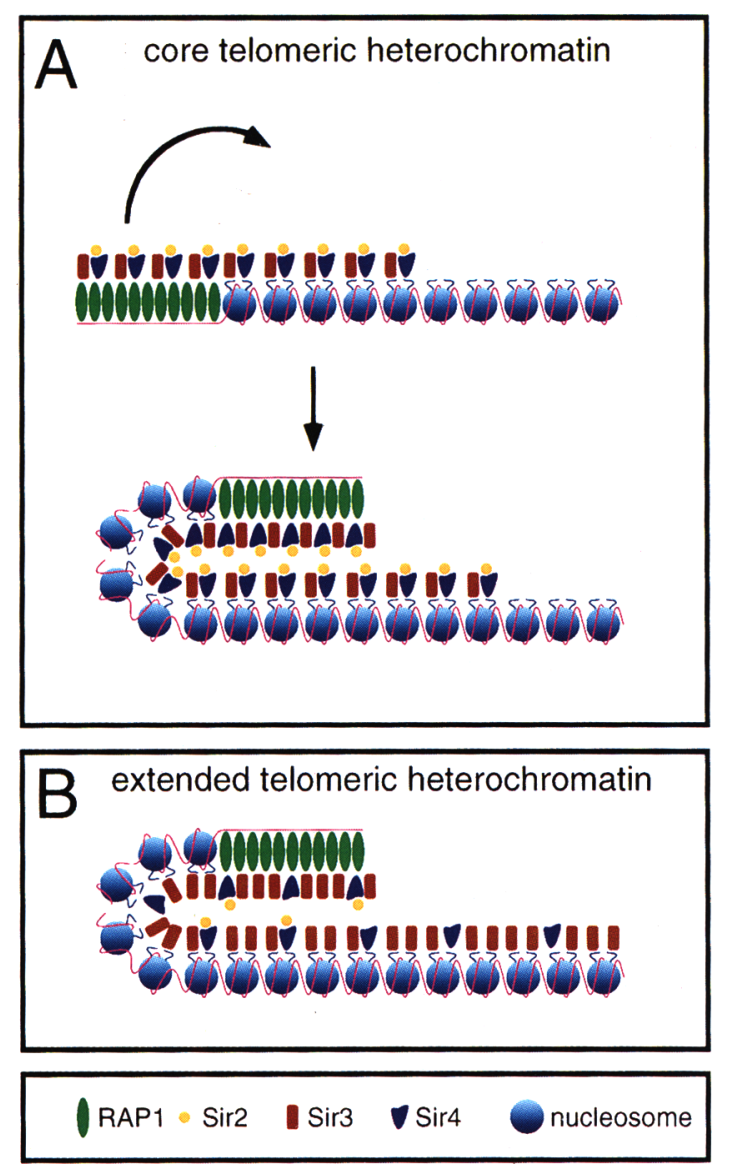

Figure 6. A model for the formation of core and extended telomeric heterochromatin. $\{A\}$ Core telomeric heterochromatin in wild-type cells: RAP1 may nucleate the binding of SIR2-SIR4SIR3 complexes at the telomeric $\mathrm{C}_{1-3} \mathrm{~A}$ repeats. SIR complexes polymerize further into adjacent chromatin by interactions with the histones $\mathrm{H} 3$ and $\mathrm{H} 4$ (upper panel). The telosome may form a loop allowing RAP1-SIR-histone complexes to associate at a number of subtelomeric regions (lower panel). Multiple homotypic and heterotypic interactions between the SIR proteins might stabilize this complex, formed of many weakly interacting partners. $(B)$ Extended telomeric heterochromatin formed upon SIR3 overexpression. Driven by higher SIR3 concentrations, SIR3-SIR3 and SIR3-histone contacts may compete successfully against interactions with other SIR proteins.
(Hecht et al. 1996), may compete successfully against SIR3 interactions with other SIR proteins. Similar effects have long been known in Drosophila, in which even a factor of two difference in concentration of certain proteins alters PEV (Locke et al. 1988). Therefore, it appears likely that the principles determined in yeast for both the initiation and spreading of heterochromatin components will be applicable to more complex eukaryotes.

\section{Materials and methods}

Yeast strains

Yeast strains are described in Table 1. All strains used in this study are derived from AYH2.8. SIR3 carrying two tandem copies of the influenza virus hemagglutinin epitope (Wilson et al. $1984)$ at the very carboxyl terminus (SIR3HA) was reintegrated into AYH2.8 resulting in strain AYH2.45. To disrupt SIR2 by homologous recombination, AYH2.45 was transformed with the plasmid pSB42 (digested with SphI and EcoRI), resulting in strain STY30. To generate the SIR4 disruption strain STY36, the ScaI-EcoRI-digested plasmid pSB45 was transformed into strain AYH2.45. Yeast shuttle vectors pRS $424(2 \mu, T R P 1$; Christian-

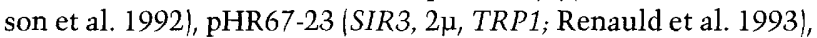

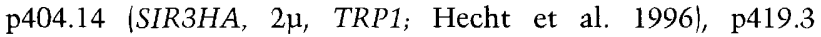
(SIR3HA, $2 \mu$, LYS2; Hecht et al. 1996) were transformed into the strains AYH2.45, AYH2.8, AYH2.38, or AYH2.46. All yeast transformations were performed following the method of Gietz et al. (1992). Gene disruptions were confirmed by Southern and Western blot analyses.

\section{Plasmid constructions}

Standard procedures were used for all DNA manipulations (Sambrook et al. 1989). Unless otherwise mentioned all clonings and transformations were carried out in E. coli host DH5 $\alpha$.

The SIR4 disruption plasmid pSB45 was generated by cloning the $2.9-\mathrm{kb} B a m \mathrm{HI}-E c o \mathrm{RI}$ fragment from pJR643 into pUC18 missing the $X b a I$ to HindIII sites in the polylinker. The SphI$B a m H I$ fragment of the resulting plasmid was replaced with the 1.0-kb SpHI-BgIII from plR643. Subsequently the TRP1 gene was inserted by blunt-end ligation into the $S p h I$ site.

To construct the SIR2 disruption plasmid pSB42, the $1.6-\mathrm{kb}$ SphI-StuI fragment from pJR68 was cloned into pUC18 and the TRP1 gene inserted into the BamHI site by blunt-end ligation.

GST fusion plasmids were obtained as follows: After cloning the NdeI-DraI fragment coding for SIR4 from the codons for amino acids 142-591 into the SmaI site of pBluescript SK (Stratagene), the fragment was excised with BamHI and EcoRI

Table 1. Yeast strains used in this study

\begin{tabular}{|c|c|c|}
\hline Strain & Genotype & Reference \\
\hline AYH2.8 & 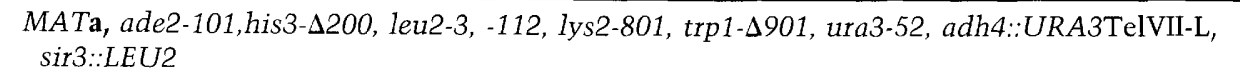 & Hecht et al. (1996) \\
\hline AYH2.45 & 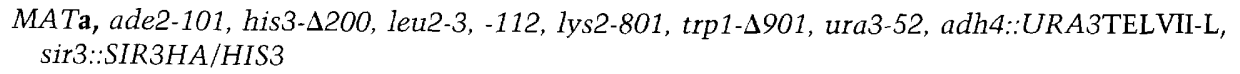 & Hecht et al. (1996) \\
\hline AYH2.38 & $\begin{array}{l}\text { MATa, ade2-101, his3-4200, leu2-3, -112, lys2-801, trp1-4901, ura3-52, adh4::URA3TelVII-L, } \\
\text { sir3::LEU2, sir4::HIS3 }\end{array}$ & Hecht et al. (1996) \\
\hline AYH2.46 & $\begin{array}{l}\text { MATa, ade2-101, his3- } 2200, \text { leu2-3, }-112, \text { lys2-801, trp1-A901, ura3-52, adh4::URA3TelVII-L, } \\
\text { Sir3::LEU2, rap1::HIS3 with p438.2 (rap1-21, CEN ARS, TRP1) }\end{array}$ & Hecht et al. (1996) \\
\hline STY30 & 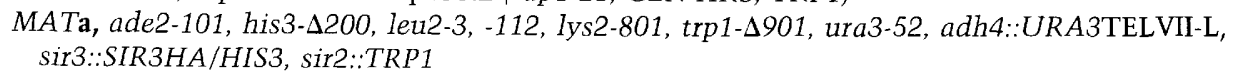 & this study \\
\hline STY36 & 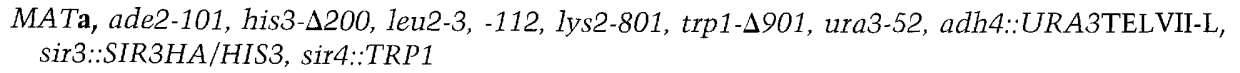 & this study \\
\hline
\end{tabular}


and subsequently introduced into pGEX2TK (Pharmacia) resulting in pKL106 (GST-SIR4N).

Plasmid pSB28 (GST-SIR4C) was obtained by cloning the PvuII fragment coding for SIR4, amino acids 1144-1358, into the SmaI site of pBluescript SK. The insert was then transferred into pGEX2TK using BamHI and EcoRI. Correct GST fusion junctions were confirmed by sequencing.

Plasmids described in Hecht et al. (1995) were used for in vitro transcription of SIR2 and SIR3.

\section{In vitro protein-binding assay}

Following the protocol of Smith and Johnson (1988) GST, GSTSIR4N, and GST-SIR4C fusions were expressed in E. coli strain BL21, purified and bound to glutathione-Sepharose 4B beads (Pharmacia) at a concentration of $10 \mu \mathrm{g}$ GST fusion protein per $10-\mu \mathrm{l}$ bed volume beads. The TNT T3-coupled reticulocyte lysate system (Promega) was used to synthesize SIR2 and SIR3 in vitro in the presence of $\left[{ }^{35} \mathrm{~S}\right]$ methionine (ICN). Binding studies were performed according to our previous protocol (Hecht et al. 1995|, with the exception that the E. coli BL21 crude extract present during the binding reaction was substituted by BSA $(100$ $\mu \mathrm{g} / \mu \mathrm{ll}$. In SIR4-SIR2 binding studies the binding buffer described by Moretti et al. (1994) was used. Proteins $20 \%$ of the input material and $40 \%$ of the eluates) were resolved by SDSpolyacrylamide gel electrophoresis (PAGE). The gels were treated with fixing solution (isopropanol to acetic acid to $\mathrm{H}_{2} \mathrm{O}$, 25:10:65) and Amplify (Amersham) for 20 min each, and dried. Proteins were visualized by fluorography.

\section{Antibodies}

Antihemagglutinin epitope monoclonal antibody (17D09) was covalently coupled to protein A-Sepharose CL-4B as described (Hecht et al. 1996). Anti-SIR3, anti-SIR4, and anti-RAP1 antibodies were as reported in Hecht et al. (1996). For the production of anti-SIR2 polyclonal antibodies the SIR2 carboxy-terminal amino acids 275-562 were fused to GST. The fusion protein was expressed in and purified from E. coli BL21 (Smith and Johnson 1988). Rabbit polyclonal antibodies to GST-SIR2 (amino acids 275-562) were produced using standard methods (Harlow and Lane 1988). Antibodies were affinity purified by binding to nitrocellulose derivatized with the GST fusion protein of relevance following the protocol of Olmsted (1981) and tested for their specificity by Western blot analyses comparing whole-cell extracts (see below) from wild-type and mutant yeast strains.

\section{Western blotting}

Proteins were fractionated on $8 \%$ SDS-polyacrylamide gels and transferred to nitrocellulose (Harlow and Lane 1988). Anti-SIR4 polyclonal antibodies were used at 1:5000, anti-SIR3 polyclonal antibodies at 1:10,000, and anti-SIR2 polyclonal antibodies at 1:3000 dilution. Protein-antibody complexes were visulized by enhanced chemiluminescence using the Amersham ECL system.

\section{Coimmunoprecipitation from yeast whole-cell extracts}

Yeast cells were grown on SD medium to a concentration of $2.0 \times 10^{7}$ cells $/ \mathrm{ml}$. Cells $(50 \mathrm{ml})$ were harvested, washed twice with TBS (20 mM Tris- $\mathrm{HCl}$ at $\mathrm{pH} 7.6,200 \mathrm{~mm} \mathrm{NaCl})$, and resuspended in $400 \mu 1$ lysis buffer $(50 \mathrm{~mm}$ HEPES-KOH at $\mathrm{pH} 7.5$, $140 \mathrm{~mm} \mathrm{NaCl}, 1 \mathrm{~mm}$ EDTA, $10 \%$ glycerol, $0.5 \%$ NP-40, $1 \mathrm{~mm}$ PMSF, $1 \mathrm{~mm}$ benzamidine, $0.25 \mathrm{~mm}$ TLCK, $50 \mu \mathrm{g} / \mathrm{ml}$ TPCK, 10 $\mu \mathrm{g} / \mathrm{ml}$ aprotinin, $20 \mu \mathrm{g} / \mathrm{ml}$ antipain, $1 \mu \mathrm{g} / \mathrm{ml}$ leupeptine, and 1 $\mu \mathrm{g} / \mathrm{ml}$ pepstatin). An equal volume of glass beads (diameter 0.5 $\mathrm{mm}$ ) was added. Breakage was achieved by vortexing on an Eppendorf shaker 5432 for $40 \mathrm{~min}$ at $4^{\circ} \mathrm{C}$. The bottom of the tube was punctured and the lysate collected by brief centrifugation. The suspension was clarified by centrifugation for $5 \mathrm{~min}$ and 15 min, respectively, in a microcentrifuge to yield the whole-cell extract. Immunopreciptiation of SIR3HA was performed as described in Heicht et al. (1996). For immunoprecipitation of SIR2, SIR4, or RAP1 the amount of antibodies needed to remove the protein quantitatively from the whole-cell extract was determined. The affinity-purified anti-SIR2, -SIR4, or -RAP1 antibodies were added to $400 \mu \mathrm{l}$ of whole-cell extract and incubated for $3 \mathrm{hr}$ at $4^{\circ} \mathrm{C}$. Unless stated otherwise DNase I (250 units) was present during immunoprecipitation. Sixty microliters of bedvolume protein A-Sepharose CL-4B beads (Pharmacia) were added, and the incubation continued for $1 \mathrm{hr}$. The immunoprecipitates were washed three times for $5 \mathrm{~min}$ with $1.4 \mathrm{ml}$ lysis buffer and subsequently resuspended in $60 \mu \mathrm{l}$ SDS-PAGE sample buffer. Thirty to forty microliters of the eluted proteins were analyzed by SDS-PAGE and Western blotting. For the DNaseI experiments $30-\mu l$ aliquots of the supernatants after immunoprecipitation were treated with $1 \mu \mathrm{g} / \mu \mathrm{l}$ proteinase $\mathrm{K}$ and the DNA was subsequently purified by phenol extraction.

\section{Immunoprecipitation from fixed whole-cell extracts}

Yeast cells were grown as above. Fifty milliliters of cells $\left(2.0 \times 10^{7}\right.$ cells $\left./ \mathrm{ml}\right)$ were cross-linked with $1 \%$ formaldehyde for $15 \mathrm{~min}$ at room temperature. Glycine was added to a final concentration of $125 \mathrm{~mm}$ and the incubation continued for $5 \mathrm{~min}$. Cells were harvested and washed twice with TBS, and breakage was performed in $400 \mu \mathrm{lFA}$-lysis buffer ( $50 \mathrm{~mm}$ HEPES-KOH at $\mathrm{pH} 7.5,140 \mathrm{~mm} \mathrm{NaCl}, 1 \mathrm{~mm}$ EDTA, $1 \%$ Triton X-100, $0.1 \%$ sodium deoxycholate, $1 \mathrm{~mm}$ PMSF, $1 \mathrm{~mm}$ benzamidine, $0.25 \mathrm{~mm}$ TLCK, $50 \mu \mathrm{g} / \mathrm{ml}$ TPCK, $10 \mu \mathrm{g} / \mathrm{ml}$ aprotinin, $20 \mu \mathrm{g} / \mathrm{ml}$ antipain, $1 \mu \mathrm{g} / \mathrm{ml}$ leupeptine, and $1 \mu \mathrm{g} / \mathrm{ml}$ pepstatin) as above. The suspension was sonicated either twice for $10 \mathrm{sec}$ each /resulting in an average fragment size of $0.5-1 \mathrm{~kb}$ ) of 14 times for $10 \mathrm{sec}$ each (resulting in an average fragment size of $0.5-0.3 \mathrm{~kb}$ ) and clarified as before. Immunoprecipitations were performed as described above (no DNaseI added). Precipitates were succesively washed for 5 min each with $1.4 \mathrm{ml}$ of FA-lysis buffer, $1.4 \mathrm{ml}$ of FA-lysis buffer $/ 500 \mathrm{mM} \mathrm{NaCl}, 1.4 \mathrm{ml}$ of $10 \mathrm{mM}$ Tris- $\mathrm{HCl}$ at $\mathrm{pH} 8.0,0.25$ $\mathrm{M} \mathrm{LiCl}, 0.5 \%$ NP-40, 0.5\% sodium deoxycholate, $1 \mathrm{mM}$ EDTA, and $1.4 \mathrm{ml}$ of TE (20 mM Tris- $\mathrm{HCl}$ at $\mathrm{pH} 8.0,1 \mathrm{~mm}$ EDTA). Finally, the samples were processed for DNA purification (Orlando and Paro 1993). From $\sim 60 \mu \mathrm{g}$ chromatin-DNA aliquot, immunoprecipitations with anti-SIR3HA, -SIR4, -SIR2, and -RAP1 antibodies yielded between 0.3 and $1.0 \mathrm{ng}$ DNA.

\section{PCR analyses of immunoprecipitated DNA}

PCR reactions were carried out in $50 \mu$ l volume with $1 / 50$ of the immunoprecipitated material, $1 / 13,500$ of the input material, and serial 2.5 -fold dilutions thereof as templates. Taq polymerase (GIBCO/BRL) and the corresponding buffer system was used. Seventy picomoles primer were added. The gene specific primers were designed as 24 mers with $\sim 50 \%$ GC content. The PCR cycles were chosen empirically, and determined by preliminary reactions with each set of oligonucleotides, and the reactions stopped before reagents were exhausted. Typically, an initial denaturation for $2 \mathrm{~min}$ at $95^{\circ} \mathrm{C}$ was followed by 25 cycles with denaturation for $30 \mathrm{sec}$ at $95^{\circ} \mathrm{C}$, annealing for $30 \mathrm{sec}$ at $55^{\circ} \mathrm{C}$, polymerization for $60 \mathrm{sec}$ at $72^{\circ} \mathrm{C}$, and a final extension for 2 min at $72^{\circ} \mathrm{C}$. PCR products were separated on $6 \%$ or $15 \%$ polyacrylamide gels and visualized with $0.1 \mathrm{mg} / \mathrm{ml}$ ethidium 
bromide. The gels were photographed using Polaroid film type 667 and type 55. Photoprocessing was performed using OFOTO (Light Source Computer Images) and NIH Image (version 1.49) software.

\section{Acknowledgments}

We are grateful to T. Christianson, D. Gottschling, A. Lustig, and J. Rine for the gift of plasmids; G. Fieser for the monoclonal antibody 17D09; and X. Chen for affinity purification of the anti-RAP1 antibody. We also wish to thank $M$. Büttner and the members of the Grunstein lab for helpful discussions, and especially X.-J. Ma and R. Mann for their critical comments of this manuscript. This work was supported by Public Health Service grant GM 42421 from the National Institutes of Health. S.S.-B. received a fellowship from the Deutsche Forschungsgemeinschaft.

The publication costs of this article were defrayed in part by payment of page charges. This article must therefore be hereby marked "advertisement" in accordance with 18 USC section 1734 solely to indicate this fact.

\section{References}

Allshire, R.C., J.P. Javerzat, N.J. Redhead, and G. Cranston. 1994. Position effect variegation at fission yeast centromeres. Cell 176: 157-169.

Aparicio, O.M., B.L. Billington, and D.E. Gottschling. 1991. Modifiers of position effect are shared between telomeric and silent mating-type loci in S. cerevisiae. Cell 66: 12791287.

Brachmann, C., J.M. Sherman, S.E. Devine, E.E. Cameron, L. Pillus, and J. Boeke. 1995. The SIR2 gene family, conserved from bacteria to humans, functions in silencing, cell cycle progression, and chromosome stability. Genes \& Dev. 9: 2888-2902.

Braunstein, M., A.B. Rose, S.G. Holmes, C.D. Allis, and J.R. Broach. 1993. Transcriptional silencing in yeast is associated with reduced nucleosome acetylation. Genes \& Dev. 7: 592604.

Buchman, A.R., W.J. Kimmerly, J. Rine, and R.D. Kornberg. 1988. Two DNA-binding factors recognize specific sequences at silencers, upstream activating sequences, autonomously replicating sequences, and telomeres in $S$. cerevisiae. Mol. Cell. Biol. 8: 210-225.

Buck, S.W. and D. Shore. 1995. Action of a RAP1 carboxy-terminal silencing domain reveals an underlying competition between HMR and telomeres in yeast. Genes \& Dev. 9: 370384.

Chien, C.-T., P.L. Bartel, R. Sternglanz, and S. Fields. 1991. The two-hybrid system: A method to identify and clone genes for proteins that interact with a protein of interest. Proc. Natl. Acad. Sci, 88: 9578-9582.

Christianson, T.W., R.S. Sikorski, M. Dante, J.H. Shero, and P. Hieter. 1992. Multifunctional yeast high-copy-number shuttle vectors. Gene 110: 119-122.

Cockell, M., F. Palladino, T. Laroche, G. Kyrion. C. Liu, A.J. Lustig, and S.M. Gasser. 1995. The carboxy termini of Sir4 and Rap1 affect Sir3 localization: Evidence for a multicomponent complex required for yeast telomeric silencing. $I$. Cell Biol. 129: 909-924.

Diffley, J.F. and B. Stillmann. 1989. Transcriptional silencing and lamins. Nature 342: 24.

Gietz, D., A. St. Jean, R.A. Woods, and R.H. Schiestl. 1992. Improved method for high efficeincy transformation of in- tact yeast cells. Nucleic Acids Res. 20:1425.

Gotta, M., T. Laroche, A. Formenton, L. Maillet, H. Shertan, and S.M. Gasser. 1996. Clustering of telomeres and their colocalization with Rap1, Sir3 and Sir4 proteins in wild-type $S$. cerevisiae. J. Cell Biol. 134: 1349-1363.

Gottlieb, S. and R.E. Esposito. 1989. A new role for a yeast transcriptional silencer gene, SIR2, in regulation of recombination in ribosomal DNA. Cell 63: 751-762.

Gottschling, D.E., O.M. Aparicio, B.L. Billington, and V.A. Zakian. 1990. Position effect at $S$. cerevisiae telomeres: Reversible repression of Pol II transcription. Cell 63: 751-762.

Hardy, C.F., L. Sussel, and D. Shore. 1992. A RAP1-interacting protein involved in transcriptional silencing and telomere length regulation. Genes \& Dev. 6: 801-814.

Harlow, E. and D. Lane. 1988. Antibodies: A laboratory manual. Cold Spring Harbor Laboratory, Cold Spring Harbor, NY.

Hecht, A., T. Laroche, S. Strahl-Bolsinger, S.M. Gasser, and M. Grunstein. 1995. Histone H3 and H4 N-termini interact with the silent informatin regulators Sir3 and Sir4: A model for the formation of heterochromatin in yeast. Cell 80: 583592.

Hecht, A., S. Strahl-Bolsinger, and M. Grunstein. 1996. Spreading of transcriptional repression by SIR3 from telomeric heterochromatin. Nature 383: 92-96.

Heitz, E. 1928. Das Heterochromatin der Moose. Jahrb. Wiss. Bot. 69: 762-818.

Henikoff, S. 1990. Position effect variegation after 60 years. Trends Genet. 6: 422-426.

Johnson, L.M., P.S. Kayne, E.S. Kahn, and M. Grunstein. 1990. Genetic evidence for an interaction between SIR3 and histone $\mathrm{H} 4$ in the repression of the silent mating loci in $S$. cerevisiae. Proc. Natl. Acad. Sci. 87: 6286-6290.

Kurtz, S. and D. Shore. 1991. RAP1 protein activates and silences transcription of mating-type genes in yeast. Genes \& Dev. 5: 616-628.

Kyrion, G., K. Liu, C. Liu, and A.J. Lustig. 1993. RAP1 and telomere structure regulate telomere position effects in $S$. cerevisiae. Genes \& Dev. 7: 1146-1159.

Laurenson, P. and J. Rine. 1992. Silencers, and heritable transcriptional states. Microbiol. Rev. 56: 543-560.

Liu, C. and A.J. Lustig. 1996. Genetic analysis of Rap1/Sir3 interactions in telomeric and HML silencing in Saccharomyces cerevisiae. Genetics 143: 81-93.

Liu, C., X. Mao, and A.J. Lustig. 1994. Mutational analysis defines a C-terminal tail domain of RAP1 essential for telomeric silencing in Saccharomyces cerevisiae. Genetics 138: 1025-1040.

Locke, J., M.A. Kotarski, and K.D. Tartof. 1988. Dosage-dependent modifiers of position effect variegation in Drosophila and a mass action model that explains their effects. Genetics 120: 181-198.

Maillet, L., C. Boscheron, M. Gotta, S. Marcand, E. Gilson, and S.M. Gasser. 1996. Evidence for silencing compartments within the yeast nucleus: A role for telomere proximity and Sir protein concentration in silencer-mediated repression. Genes \& Dev. 10: 1796-1811.

Marshall, M., D. Mahoney, A. Rose, J.B. Hicks, and J.R. Broach. 1987. Functional domains of SIR4, a gene required for position effect regulation in $S$. cerevisiae. Mol. Cell. Biol. 7: 4441-4452.

Moazed, D. and A.D. Johnson. 1996. A deubiquinating enzyme interacts with SIR4 and regulates silencing in S. cerevisiae. Cell 86: 667-677.

Moretti, P., K. Freeman, L. Coodly, and D. Shore. 1994. Evidence that a complex of SIR proteins interacts with the si- 
lencer and telomere binding protein RAP1. Genes \& Dev. 8: $2257-2269$.

Olmsted, J.B. 1981. Affinity purification of antibodies from diazotized paper blots of heterogeneous protein samples. I. Biol. Chem. 256: 11955-11957.

Olson, M.V. 1991. Genome structure and organization in Saccharomyces cerevisiae. In The molecular and cellular biology of the yeast Saccharomyces (ed. J.R. Broach, J.R. Pringel, and E.W. Jones|, Vol. 1, pp. 1-39, Cold Spring Harbor Laboratory Press, Cold Spring Harbor, NY.

Orlando, V. and R. Paro. 1993. Mapping polycomb-repressed domains in the bithorax complex using in vivo formaldehyde cross-linked chromatin. Cell 75: 1187-1198.

Palladino, F., T. Laroche, E. Gilson, A. Axelrod, L. Pillus, and S.M. Gasser. 1993. SIR3 and SIR4 proteins are required for the positioning and integrity of yeast telomeres. Cell 75: 543-555.

Renauld, H., O.M. Aparicio, P.D. Zierath, B.L. Billington, S.K. Chhablani, and D.E. Gottschling. 1993. Silent domains are assembled continuously from the telomere and are defined by promoter distance and strength, and by SIR3 dosage. Genes \& Dev. 7: 1133-1145.

Sambrook, J., E.F. Fritsh, and T. Maniatis. 1989. Molecular cloning: A laboratory manual. Cold Spring Harbor Laboratory Press, Cold Spring Harbor, NY.

Shore, D. and K. Nasmyth. 1987. Purification and cloning of a DNA binding protein from yeast that binds to both silencer and activator elements. Cell 51: 721-732.

Smith, D.B. and K.S. Johnson. 1988. Single-step purification of polypeptides expressed in E. coli as fusions with glutathione S-transferase. Gene 67: 31-40.

Thompson, J.S., A. Hecht, and M. Grunstein. 1993. Histones and the regulation of heterochromatin in yeast. Cold Spring Harbor Symp. Quant. Biol. 58: 247-256.

Thompson, J.S., L.M. Johnson, and M. Grunstein. 1994a. Specific repression of the yeast silent mating type locus $H M R$ by an adjacent telomere. Mol. Biol. Cell 14: 446-455.

Thompson, J.S., X. Ling, and M. Grunstein. 1994b. The histone $\mathrm{H} 3$ amino terminus is required for both telomeric and silent mating locus repression in yeast. Nature 369: 245-247.

Weiler, K.S. and B.T. Wakimoto. 1995. Heterochromatin and gene expression in Drosophila. Annu. Rev. Genet. 29: 577 605.

Wilson, I.A., H.L. Niman, R.A. Houghten, A.R. Cherenson, M.L. COnnolly, and R.A. Lerner. 1984. The structure of an antigenic determinant in a protein. Cell 37: 767-778.

Wright, J.H., D.E. Gottschling, and V.A. Zakian, 1992. Saccharomyces telomeres assume a non-nucleosomal chromatin structure. Genes \& Dev. 6: 197-210. 


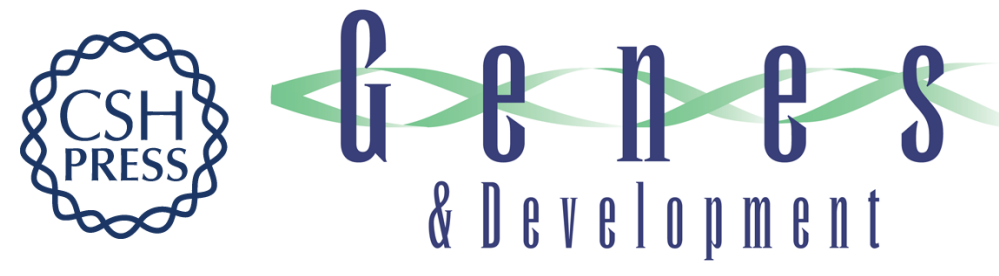

\section{SIR2 and SIR4 interactions differ in core and extended telomeric heterochromatin in yeast.}

S Strahl-Bolsinger, A Hecht, K Luo, et al.

Genes Dev. 1997, 11:

Access the most recent version at doi:10.1101/gad.11.1.83

References This article cites 42 articles, 21 of which can be accessed free at:

http://genesdev.cshlp.org/content/11/1/83.full.html\#ref-list-1

License

Email Alerting Receive free email alerts when new articles cite this article - sign up in the box at the top Service right corner of the article or click here.

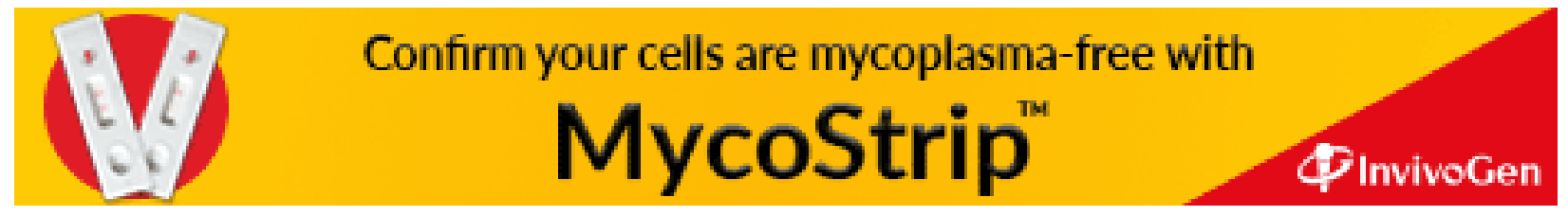

